\title{
Modeling the bending of the blade windturbine Using Finite Element Analysis.
}

\author{
Ed-dokkali Imane ${ }^{1}$, El bakkali Larbi ${ }^{1}$ \\ ${ }^{l}$ Physical, Research Team Modeling and Simulation of Mechanical Systems, Sciences Faculty, Abdelmalek \\ Essaâdi University, Tétouan - Morocco
}

\begin{abstract}
The blade in its movement undergoes three types of deformation: longitudinal (bending), lateral and twisting, of interest for the bending mode since it appears the most important mode. The approach used for modeling of blade element bending is done by the finite element method of discretizing the blade beam element, each with two degrees of nodal freedoms. Solving the equation of motion based on the MEF is obtained by the application of modal analysis which consists of obtaining from the eigenmodes and system natural frequencies that will be used in the study of forced motion due to aerodynamic loads using the modal superposition method. Theory of the airfoil member is used for the evaluation of aerodynamic loads of dividing the blade in a series of elements (airfoils) that operate independently.
\end{abstract}

Keywords: Bending mode, centrifugal effect, inertial effect, Finite element method, modal analysis, theory of the aerodynamic profile element, aerodynamic loadsn.

\section{Introduction}

Wind energy is one of the oldest sources of energy used. The wind turbine blades are the most flexible part of this machine, and modal behavior has a great influence on the total dynamic. Recently, more attention has been paid to modal analysis. This is reflected by the number of experimental and numerical studies conducted on this subject The calculation of frequencies and bending normal modes of a rotating blade is a thorny problem because of the complexity of the equation that governs its movement .In fact, this equation has no exact solution. In addition, it is characterized by complex boundary conditions that hinder any numerical solution A modeling of finite element is applied to a complex blade shape using Ansys software.

In this paper, the resolution of the equation of bending motion of the blade is solved by the finite element method using the Matlab code. The method involves the blade in small discrete beam elements, using the principle of the Galerkin residual method, which has applied the equation of motion. A modal analysis system , is carried out to obtain eigenvector and natural frequency of blade bending. The use of these results helps to study the equation of bending forces . The blade bending comes from the normal stress in this mode, the curvature is counted. To obtain responsible aerodynamic forces in bending mode, we use axial momentum theory. This theory is made to divide the blade in a series of elements (airfoils) that operate independently. The interaction between tubes' flow corresponding to the neighboring elements is neglected. Thus, it is possible to obtain independently the forces of drag and lift applied to each element, regardless of the flow in the adjacent elements .

\section{Formulation}

The wind turbine blade is treated as an elastic beam rotating with a constant angular velocity. The blade is treated as a Bernoulli beam clamped-free, vibrating right angles, which studies the elastic axis coinciding with its neutral axis supposedly. The blade is subject to the aerodynamic force. The efforts applied to the blade element are illustrated in Fig.1. The application of second law of Newton leads to an equation according to the three main axes $\mathrm{x}, \mathrm{y}$ and $\mathrm{z}$, which are attached to the undeformed blade . $\mathrm{X}$ is coincided with the axis elastic; $\mathrm{z}$ is the axis of rotation of the blade.

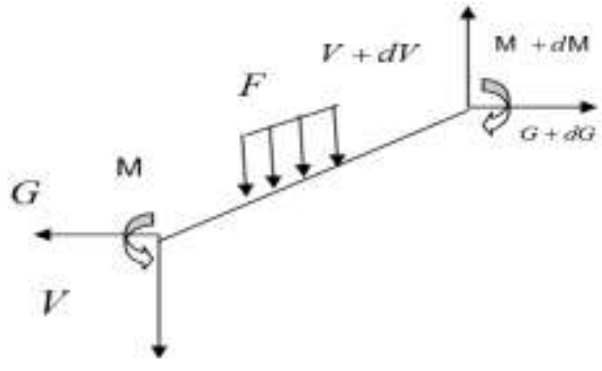

Fig.1: forces on a blade element. 


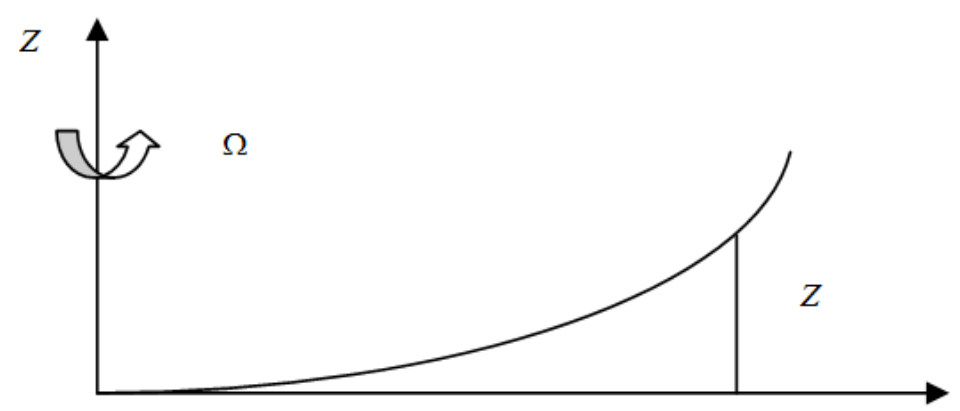

$x$

Fig.2: Deflexion of the blade

- Projection along the $\mathrm{x}$ axis:

$d G=-m \Omega^{2} r d r$

-Projection along the y axis:

$d V-F d r=m \frac{\partial^{2} Z}{\partial t^{2}} d r$

Note: Considering the weight of the element is neglected to the shear force $\mathrm{V}$

- Projection along the $\mathrm{z}$ axis:

$\frac{\partial \mathrm{M}}{\partial r}=G \frac{\partial Z}{\partial r}+V+\rho I \ddot{\theta}_{z}$

Beam Theory allows us to write the relationship between the bending moment and the shear force, so the expression of the moment is:

$$
\mathrm{M}=E I_{y} \frac{\partial^{2} Z}{\partial r^{2}}
$$

The coupling of three equations allows us to have the differential equation of 4 th order:

$E I_{y} \frac{\partial^{4} Z}{\partial r^{4}}-G \frac{\partial^{2} Z}{\partial r^{2}}+m \frac{\partial^{2} Z}{\partial t^{2}}=\frac{\partial F}{\partial r}$

\section{Aerodynamic Loads}

It is assumed that the aerodynamic load acting on a blade element is uniform as shown in Fig1.

1- In the non- deformed configuration

The theory applied to the blade member involves dividing the blade in a series of elements (airfoils) that operate independently. This mean that the interaction between the flow tubes corresponding to the adjacent elements is neglected. Thus, it is possible to obtain independently the forces of drag and lift applied to each element, regardless of the flow in the adjacent elements [2], [3]. The pressure field on a profile gives rise to an elementary aerodynamic force. It comprises of two elementary forces (Fig.3).

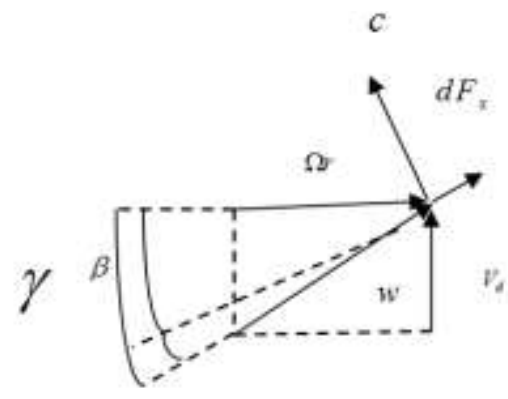

Direction of

speed

Fig.3: Section of an element of the blade to a radius $r$ 


$$
\begin{aligned}
& d F_{x}=0.5 \rho_{a} w^{2} C_{x}(\beta) c d r \\
& d F_{z}=0.5 \rho_{a} w^{2} C_{z}(\beta) c d r
\end{aligned}
$$

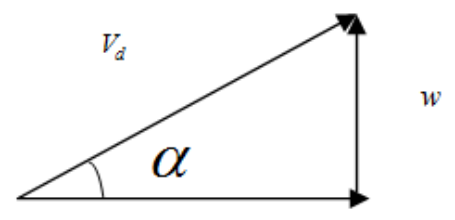

$\Omega r$

Fig.4: Speed triangle

$\beta=\alpha-\gamma$

$\alpha=\arctan \left(\frac{V_{d}}{\Omega r}\right)$

$C_{x}$ and $C_{z}$ are coefficients of drag and lift, respectively, depend on coefficients $\beta$. These profiles are obtained from numerical simulations or tests [7]. Fig.5 and Fig.6 show the aerodynamic characteristics Profile NACA2412 [7]. $d F_{n}=d F_{x} \sin \alpha+d F_{z} \cos \alpha$

Only the normal component occurs in the case of bending.

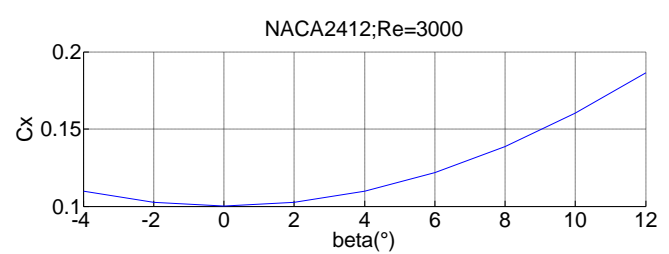

Fig.5: Coefficient of the drag for NACA 2412; $=3000$

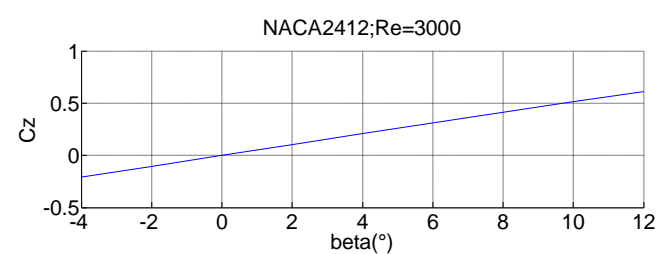

Fig.6: Coefficient of the lift for NACA 2412; $=3000$

2- In the deformed configuration

Deformation of the blade bending at $\mathrm{z}$ axis introduced a quantity $\mathrm{dz}$ that applies a variation in the angle of incidence, in this case $\beta$ becomes:

$\beta^{\prime}=\alpha-\gamma-u$

With the expression of $u$ (see fig.7)

$u=\arctan \left(\frac{d z}{d x}\right)$

The expressions found in the case of the undeformed blade will be replaced by the following expression:

$$
w^{2}=\left(v^{2}+(\Omega r)^{2}\right) \cos ^{2} u
$$

Forces drags and lift become as follow:

$$
\begin{aligned}
& d F_{z}=0.5 \rho_{a} w^{\prime 2} C_{z}\left(\beta^{\prime}\right) c d r \\
& d F_{x}=0.5 \rho_{a} w^{\prime 2} C_{x}\left(\beta^{\prime}\right) c d r
\end{aligned}
$$




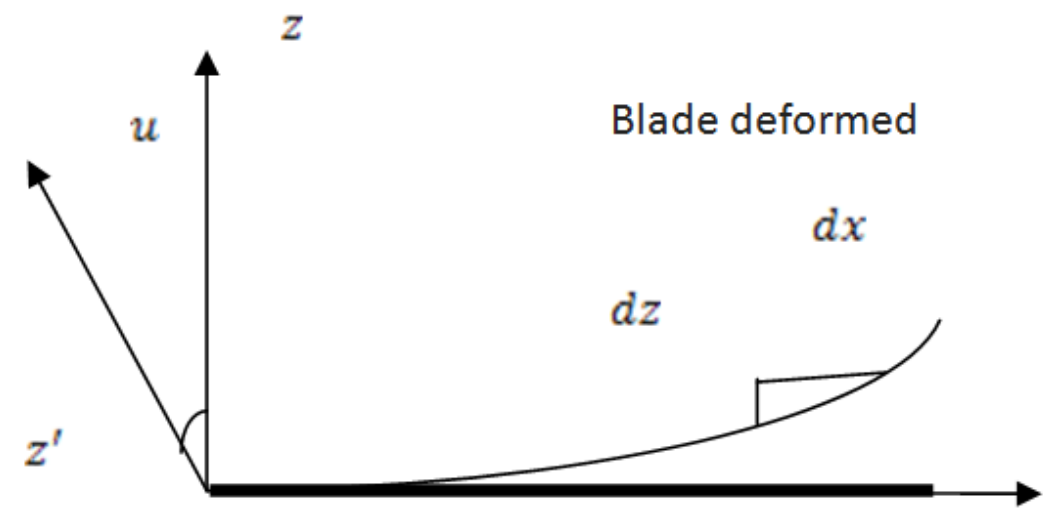

\section{Blade undeformed}

Fig.7: Forces at the blade deformed

\section{Finite Element Method}

Analysis by the finite element method [6], [10] is useful for the space discretization of the equilibrium equation of motion. The blade is divided into beam elements. Each element consists of two nodes, with two degrees of freedom in each node and denoted $v$ and $\theta$ as shown in fig.8. The distribution of $\mathrm{z}$ is represented by the nodal displacement by using the interpolation function:

$Z(x)=\{H\}\left\{\Delta_{i}\right\}$

$\{H\}$ The interpolation vector that expresses:

$H=\left[\begin{array}{l}H_{1} \\ H_{2} \\ H_{3} \\ H_{4}\end{array}\right]$

And the vector of degrees of freedom of an element is defined as:

$$
\left\{\Delta_{i}\right\}=\left\{\begin{array}{c}
v_{i} \\
\theta_{i} \\
v_{j} \\
\theta_{j}
\end{array}\right\}
$$

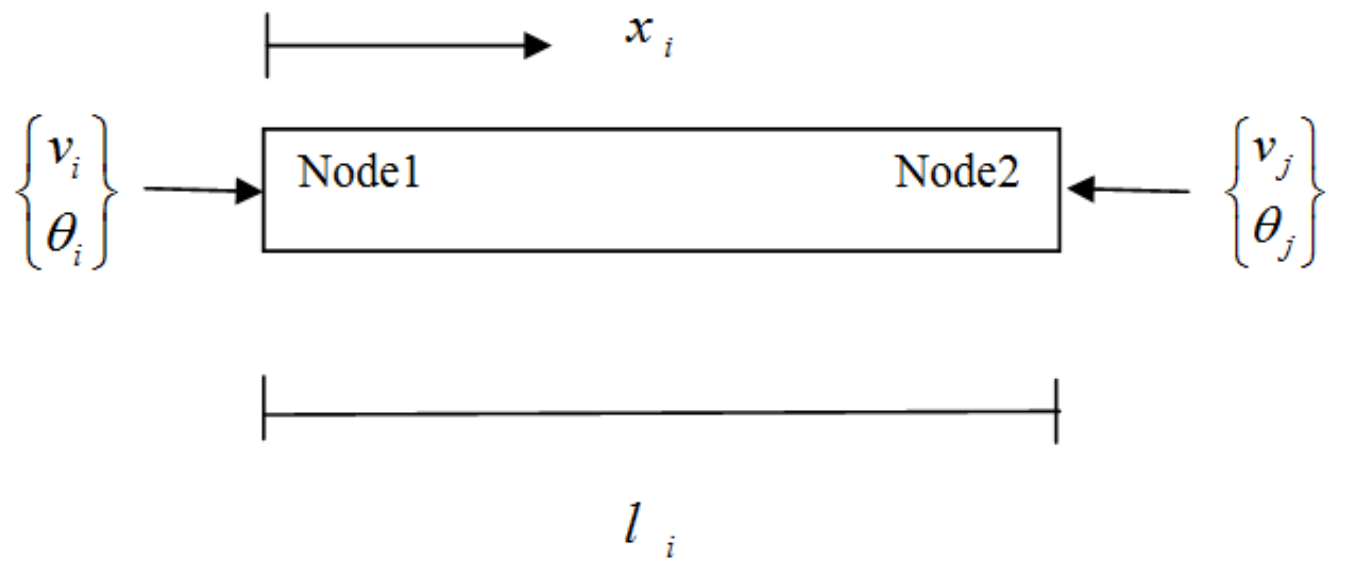

Fig.8: A finite element showing nodal degrees of freedom. 
The interpolation function of Equation (17) is the Hermit polynomial and is defined as follows:

$$
\{H\}=\left\{\begin{array}{c}
1-3\left(\frac{x}{l}\right)^{2}+2\left(\frac{x}{l}\right)^{3} \\
\left(\frac{x}{l}\right)-2\left(\frac{x}{l}\right)^{2}+\left(\frac{x}{l}\right)^{3} \\
3\left(\frac{x}{l}\right)^{2}-2\left(\frac{x}{l}\right)^{3} \\
-\left(\frac{x}{l}\right)^{2}-\left(\frac{x}{l}\right)^{3}
\end{array}\right\}
$$

Substituting the formula (16) in equation (5) and applying the weighted residue Galerkin to this equation (5) we obtain the equation of the following element:

$M_{e} \ddot{\Delta}_{i}+K_{e} \Delta_{i}=F_{e}$

With

$$
K_{e}=K_{e 1}+K_{e G}
$$

The global matrices of the system are obtained by assembling the elementary matrices and the assembly procedure is shown in fig. 9
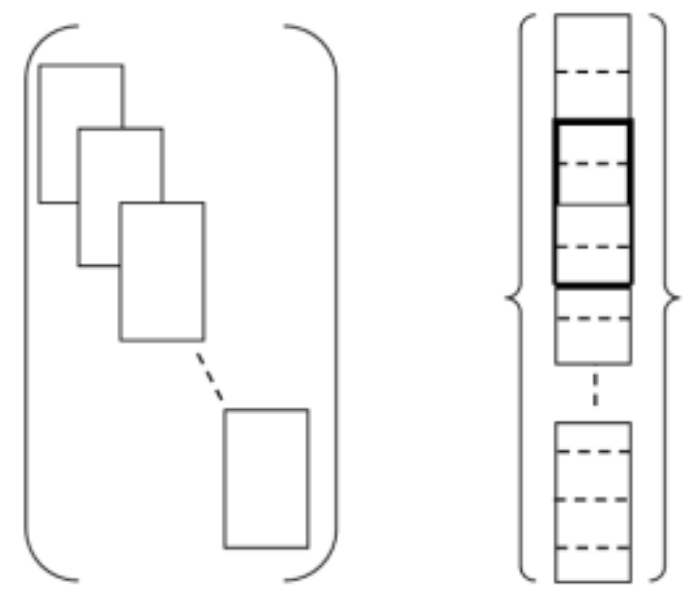

Fig.9: The assembly process of elementary matrices and elementary vectors forces.

There is then the equation of motion of the blade in the absence of damping by an equation of the same form as that of an element [10].

$$
M \ddot{\Delta}+K \Delta=F
$$

\section{Boundary Conditions}

In this case, we studied a free beam. This blade condition embedding one extremity involves the cancellation of the potential and kinetic energies. At this point, this is equivalent to removing 2 rows and 2 columns of the mass and stiffness matrices in the overall equation of the system.

\section{- Solution Procedure :}

- Free motion Study

The motion is free when the blade is not required by external forces, which means that equation (5) becomes:

$M \ddot{\Delta}+K \Delta=0$

In this case the solution takes the form: 


$$
\Delta(t)=x \sin (\omega t+\varphi)
$$

Substituting (24) in the equation (23) we get:

$\left(K-\omega_{i}^{2} M\right) x_{i}=0$

- Property eigenmodes

$$
\begin{aligned}
& X_{m}^{T} M X_{n}=\delta_{m n} \\
& X_{m}^{T} K X_{n}=\delta_{m n}
\end{aligned}
$$

These orthogonality property modes will operate with the study of forced motions.

- The Study of forced motions:

The equation is solved with modal superposition method. In order to decouple the equations of this system, we use the orthogonality property of the eigenmodes. It is therefore interesting to rewrite this equation in the base of the natural modes [14]. The displacement $\Delta$ is written in matrix form:

$$
\Delta(\mathrm{t})=\mathrm{P} \varphi(\mathrm{t})
$$

Equation (20) allows having a $2 \mathrm{n}$ equation each equation represents a system with one degree of freedom:

$$
\ddot{\varphi}_{k}(t)+\omega_{k}^{2} \varphi_{k}(t)=C_{k}
$$

Note that the terms of the first part of the equation for a given mode are independent of other modes, while the second member (aerodynamic load) depends on all modes.

The solution of equation (29) is:

$\varphi_{k}(t)=\frac{C_{k}}{\omega_{k}^{2}}\left(1-\cos \left(\omega_{k} t\right)\right)+\varphi_{0} \cos \left(\omega_{k} t\right)+\frac{\dot{\varphi}_{0}}{\omega_{k}} \sin \left(\omega_{k} t\right)$

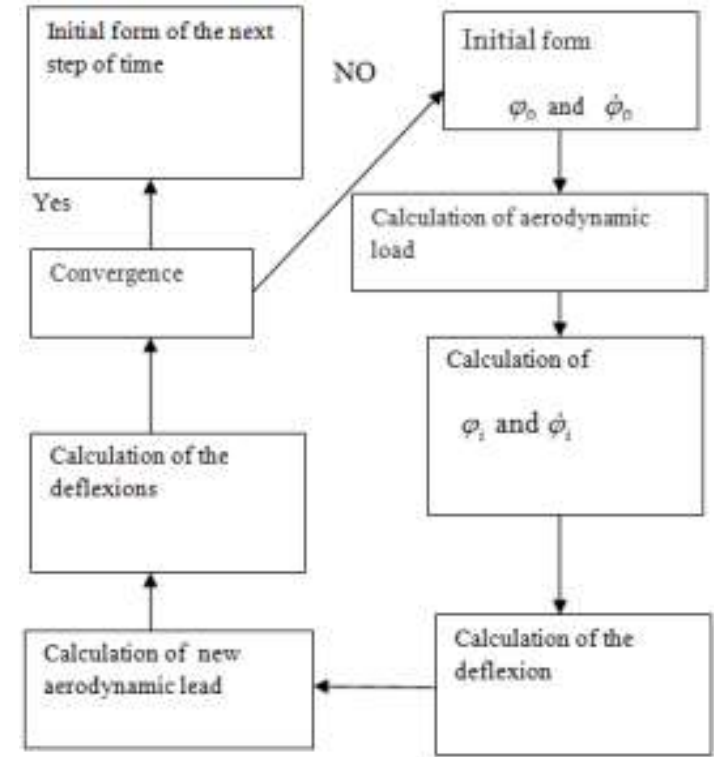

Fig.10: The flowchart summarizing the method for solving equation (22)

Features used:

\section{Results And Discussion}

NACA 2414 [8]

\begin{tabular}{|l|l|}
\hline$E$ & $5.8 .10^{9} N / m^{2}$ \\
\hline$I$ & $9.5 .10^{-7} m^{4}$ \\
\hline$L$ & $8 m$ \\
\hline$c$ & $0.4 m$ \\
\hline$\lambda$ & 8 \\
\hline$m$ & $6.69 \mathrm{~kg} / \mathrm{m}$ \\
\hline
\end{tabular}




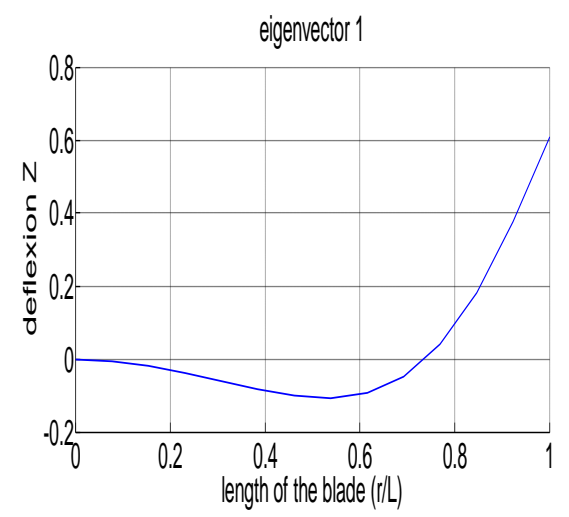

Fig.11 : eigenmode 1

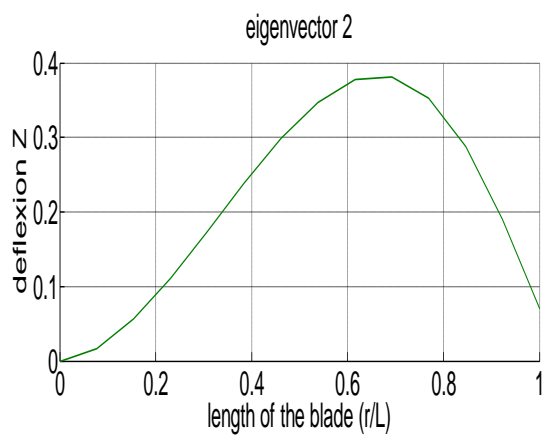

Fig.12: eigenmode2

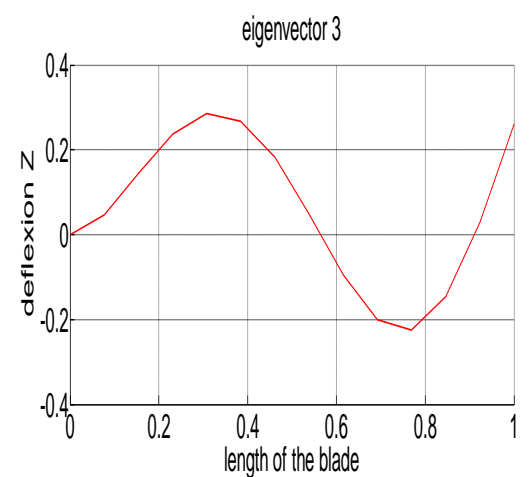

Fig.13: Eigenmode 3

Of all the mechanical vibration problems, the lowest frequency is the most interesting, for this reason, the first three modes are taken into account.

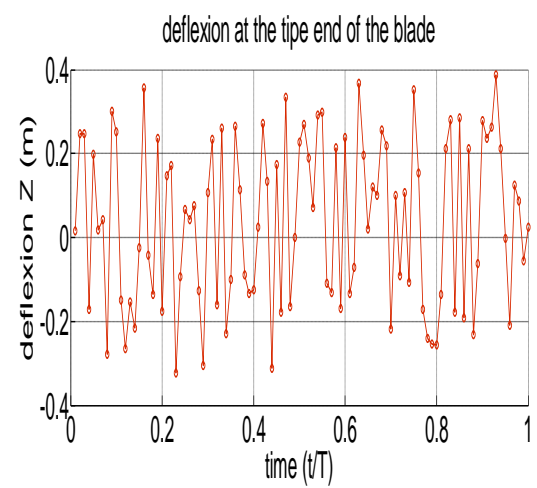

Fig.14: The defluxion of the tip end of the blade $(=12 \mathrm{rad} / \mathrm{s})$

We can see that the defluxion maximal of the tip end of the blade for $\Omega=12 \mathrm{rad} / \mathrm{s}$ is $0.3875 \mathrm{~m}$ 


\section{Conclusion}

In this work, we intended to develop a mathematical model of analysis, so a study of vibration of wind turbine blades subjected to the aerodynamic loads was carried. This allows us to calculate the slowness of the blades and to estimate their lifetime.

\section{Nomenclature:}

$t$ : Time

$G$ : Centrifugal force

$E$ : Young's modulus

$I:$ Blade cross section moments of inertia

$d F_{x}$ : Elementary drag force

$d F_{z}$ : Elementary lift force

$d F_{n}$ : Elementary normal force up to $\mathrm{x}$

$C_{x}$ : Blade-section drag coefficient

$C_{z}$ : Blade-section lift coefficient

$\Omega R$ : Rotation speed of the blade

$\beta$ : Pitch angle

$\gamma:$ Twist angle

$\alpha$ : Angle of attack

$M:$ The global mass of matrix

$K$ : The global stiffness matrix

$F$ : The global force vector acting on the entire blade

$c$ : Blade chord

$\Delta_{i}$ : Vector of an element's nodal displacement

$\{H\}$ : Vector interpolation

$\mathrm{V}:$ Shear force

$\mathrm{M}$ : Bending moment

$w$ : Blade section resultant air velocity

$v$ : Elastic displacements in the $\mathrm{z}$ directions

$\theta$ : Rotation of the node in the y directions

$x, y, z$ : Undeformed blade coordinates

$\rho_{a}$ : Density of air

$\rho$ : Density of the element of the blade

$\{\Delta\}$ : Vector of global degrees of freedom

$\Omega$ : Rotor blade angular velocity

$m$ : Mass per unit length of blade

$\varphi:$ Test function

$V_{d}:$ Air velocity, $\mathrm{m} / \mathrm{sec}$

$K_{e}:$ Stiffness matrix

$P$ : Eigen vector

$\delta$ : The Kronecker symbol

$K_{e 1}$ : Stiffness matrix due to deformation 
$K_{G}:$ Stiffness matrix due to centrifugal force

$Z$ : Deflexion of bending of the blade

$r$ : Distance from the axis of rotation

$L$ : The length of the blade

$l$ : Length of an element of the blade

$\lambda$ :specific speed

\section{Journal Papers:}

\section{References}

[1]. Wang, Z. J. (2008). Aerodynamic efficiency of flapping flight: analysis of a two-stroke model, Journal of Experimental Biology, 211(2), 2008, 234-238

\section{Books:}

[2]. $\quad$ R.E. Moore, Interval analysis (Englewood Cliffs, NJ: Prentice-Hall, 1966

[3]. Note that the title of the book is in lower case letters and italicized. There is no comma following the title. Place of publication and publisher are given

[4]. PAULTRE, P,Dynamique des structures,Lavoisier, first edition, 2005

[5]. Delaplace, A., Gatuingt, F, \& Ragueneau, F.,Aide-mémoire de mécanique des structures-Résistance des matériaux: Résistance des matériaux. Dunod, 2008.

\section{Theses:}

[6]. Chellil A, Indentification et modélisation par élément fini des charge dynamique du rotor principale d'hélicoptère , Université de boumerdes, Faculté des sciences de l'Ingénieur, 2008

[7]. Mahri Z.L, Etude dynamique et optimisation des pales d'un aerogenerateur, Université Mentouri Constantine, Faculté des sciences de l'Ingénieur.2007

[8]. Chelirem T,Etude dynamique d'une pale d'éolienne effilée, Mentouri Constantine, Faculté des sciences exact,2010

[9]. Samper.S.,comportement dynamique de poutre composite préchargées : Modélisation par éléments finis, Université Paule Sabatier Toulouse III, 1994 